\title{
Relevance of erythrocyte sedimentation rate and C-reactive protein in patients with active uveitis
}

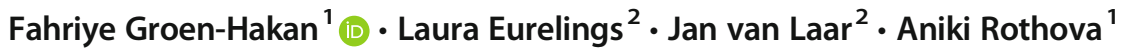

Received: 8 May 2018 /Revised: 15 October 2018 / Accepted: 25 October 2018 /Published online: 8 November 2018

(C) The Author(s) 2018

\begin{abstract}
Purpose To relate erythrocyte sedimentation rates (ESR) and C-reactive protein (CRP) values to different uveitis entisties. Methods A retrospective study of patients with a first episode of active uveitis visiting the Erasmus University Medical Center, uveitis clinic, Rotterdam, the Netherlands, was performed. Levels of ESR and CRP were determined within 2 weeks and 1 week after onset of uveitis, respectively. Uveitis had to be of unknown origin at that moment. The specific etiologic groups were related to ESR and CRP values.

Results The majority of patients with uveitis had ESR and/or CRP values within the normal limits and no association of ESR and/ or CRP with the specific cause of uveitis was observed. However, elevation of ESR $\geq 60 \mathrm{~mm} / \mathrm{h}$ and/or CRP $\geq 60 \mathrm{mg} / \mathrm{L}$ was mostly seen in patients with systemic immune-mediated diseases $(8 / 59,14 \%$ of all with immune-mediated diseases) or systemic infectious causes (7/38, 18\% of all infectious uveitis). Patients with ocular toxoplasmosis typically exhibited normal ESR and CRP $(9 / 11,82 \%)$ while patients with endogenous endophthalmitis had elevated ESR and/or CRP in 6/7, 86\%. Sarcoidosisassociated uveitis showed predominantly elevated ESR (13/24, 54\%; range 20-59 mm/h in 11/13, 85\%). Human immunodeficiency virus-positive patients had more often elevated ESR values when compared to the remainder of patients $(9 / 11,82 \%$ vs. $64 / 163,39 \%, 18 \%, P=0.009)$. The cause of uveitis was established in $19 / 20(95 \%)$ of patients with $\mathrm{ESR} \geq 60 \mathrm{~mm} / \mathrm{h}$ and/or CRP $\geq 60 \mathrm{mg} / \mathrm{L}$.

Conclusions The majority of patients with first attack of uveitis had ESR and CRP within the normal limits. Elevated levels of ESR and CRP reflected systemic involvement and high levels of both values were associated with established uveitis cause.
\end{abstract}

Keywords Erythrocyte sedimentation rate $\cdot$ C-reactive protein $\cdot$ Uveitis $\cdot$ Diagnostics

\section{Introduction}

Uveitis is an intraocular inflammation of multiple causes, which may result in permanent visual loss [1-4]. Erythrocyte sedimentation rate (ESR) and C-reactive protein

Electronic supplementary material The online version of this article (https://doi.org/10.1007/s00417-018-4174-7) contains supplementary material, which is available to authorized users.

Fahriye Groen-Hakan

f.groen@erasmusmc.nl

1 Department of Ophthalmology, Erasmus Medical Center, Erasmus University, Rotterdam, the Netherlands

2 Department of Internal Medicine and Department of Immunology, Erasmus Medical Center, Erasmus University, Rotterdam, the Netherlands
(CRP), both non-specific markers of inflammation, are usually included in the initial diagnostic workup. However, the clinical value of these parameters in the adult uveitis population is not known. Earlier investigations showed that ESR and CRP are within the normal range in a majority of patients with anterior uveitis [5]. In contrast, a recent report on juvenile idiopathic arthritis (JIA)-associated uveitis in a pediatric population showed that elevated ESR predicted the development of uveitis in patients with JIA [6]. However, it remains debatable whether ESR and CRP have any diagnostic value in evaluation of uveitis in adult patients having a first uveitis attack of unexplained origin.

Herein, we investigate the values of ESR and CRP during the first episode of active uveitis, determined within a short period after the onset in adult patients and relate the results to specific etiologic categories and clinical characteristics of uveitis. 


\section{Materials and methods}

The Strengthening the Reporting of Observational studies in Epidemiology (STROBE) guidelines were used to ensure the reporting of this observational study and this study followed the Tenets of the Declaration of Helsinki [7].

We conducted a retrospective cross-sectional study at the ophthalmology department of the Erasmus Medical Center (Rotterdam, the Netherlands). All medical records of patients referred with new uveitis of unknown origin investigated between 2010 and 2017 were reviewed and 174 patients were identified who fulfilled our inclusion criteria. The onset of uveitis was defined as the first time active inflammation was documented by an ophthalmologist. ESR had to be determined $\leq 2$ weeks and CRP values $\leq 1$ week after the onset uveitis (as ESR normalizes within weeks and CRP levels within 7 days after resolution of tissue injury) [8]. Exclusion criteria included age less than 18 years and patients with first mild anterior uveitis episode as these patients do not undergo diagnostic screening according to our guidelines. The ESR and CRP values were arbitrarily stratified to three subgroups (normal ESR $<20 \mathrm{~mm} / \mathrm{h}$, elevated ESR between 20 and $60 \mathrm{~mm} / \mathrm{h}$, highly elevated ESR $\geq 60 \mathrm{~mm} / \mathrm{h}$ and normal CRP $<10 \mathrm{mg} / \mathrm{L}$ (as defined in our laboratory), elevated CRP between 10 and $60 \mathrm{mg} / \mathrm{L}$ and highly elevated CRP $\geq 60 \mathrm{mg} / \mathrm{L}$ ).

The following data were extracted from patients' records: age, gender, localization of uveitis, laterality, and human immunodeficiency virus (HIV) status. All immunosuppressive medications as well as co-morbidities were registered. Definitive anatomical classification was determined according to the Standardization of Uveitis Nomenclature (SUN) Working Group, by reviewing the whole follow-up period [9].

The cause of uveitis was determined after the diagnostic examinations were completed. The diagnosis of definitive ocular sarcoidosis was given to patients that had histologically proven evidence and in all other cases; the criteria from the International Workshop on Ocular Sarcoidosis (IWOS) were used [10]. For the diagnosis of tuberculosis (TB)-associated uveitis, a positive culture for mycobacteria in any fluid/tissue sample was needed. Patients with a positive tuberculin skin test (Mantoux test) or interferon gamma release assay (IGRA) test with otherwise unexplained uveitis and no other indications of active tuberculosis were labeled as of unknown origin. All other specific diagnoses were performed according to current diagnostic criteria [10-16].

All statistical analyses were performed using SPSS software (version 22.0, Chicago, IL, USA) and a $P$ value of $<$ 0.05 was considered statistically significant. Specific groups were categorized as mentioned above and compared with each other according to gender, anatomical localization of uveitis, age, and etiology. Continuous variables were described by mean and range, categorical variables with proportions, and compared using the Mann-Whitney $U$ test. Categorical variables were compared using the chi-square test or Fisher's exact test.

\section{Results}

The results of ESR and CRP measurements are shown in Table 1. Specific diagnoses in our cohort are depicted in the Supplemental Table. A majority of patients was diagnosed with associated non-infectious systemic diseases (59/174, $34 \%)$ and had non-anterior uveitis $(141 / 174,81 \%)$. Slight female preponderance was observed $(96 / 174,55 \%)$.

Immunosuppressive medication (required for other causes than uveitis) was used by $17 / 174,10 \%$ patients. Patients suffering from diabetes mellitus (DM) and patients using immunosuppressive medication more often had elevated ESR values ( $P=0.018$ for both, chi-square test), compared to the remainder of patients. No significant differences in ESR and CRP levels were found for gender, race, localization, or laterality of uveitis (all $P$ values $>0.05$, chi-square test). Furthermore, elevated values of ESR and/or CRP were not significantly associated with any of the etiologic categories.

Concordance and discrepancies between ESR and CRP are depicted in Table 2. A majority of patients had both ESR and CRP values within the normal limits $(91 / 174,52 \%)$. Elevation of only one of the parameters was seen in 50/174, $29 \%$. Elevated levels of both parameters were found in $33 / 174$, $19 \%$ patients.

The median ESR and CRP of patients with uveitis of established cause were higher than the median ESR and CRP of patients with unknown uveitis $(17.0 \mathrm{~mm} / \mathrm{h}$, range $1-$ $120 \mathrm{~mm} / \mathrm{h}$ vs. $11.0 \mathrm{~mm} / \mathrm{h}$, range $1-140 \mathrm{~mm} / \mathrm{h}$ for ESR and $3.4 \mathrm{mg} / \mathrm{L}$, range $0.4-262.0 \mathrm{mg} / \mathrm{L}$ vs. $1.9 \mathrm{mg} / \mathrm{L}$, range 0.3 $229.0 \mathrm{mg} / \mathrm{L}$ for CRP; $P=0.015$ for both, Mann-Whitney $U$ test).

Out of 20 patients with either ESR $\geq 60 \mathrm{~mm} / \mathrm{h}$ and/or CRP $\geq 60 \mathrm{mg} / \mathrm{L}$, the cause of uveitis could be determined in 19/20. Fifteen had either non-infectious systemic disease or systemic infection, which was also a cause of uveitis (Table 3). The remaining five patients had uveitis limited to the eye, but had a concurrent systemic disorder, which explained their highly elevated ESR and/or CRP but was not related to the cause of uveitis (such as multiple myeloma in a patient with infectious uveitis).

A majority of patients with infectious uveitis had ESR and CRP values within the normal limits $(17 / 38,45 \%)$ or only ESR $\geq 20 \mathrm{~mm} / \mathrm{h}(14 / 38,37 \%)$, see Table 2 . Discrepant results were more often noted in this group $(17 / 38,45 \%$ vs. $33 / 136$, $24 \% P=0.024$, chi-square test). Patients with toxoplasmosis exhibited normal ESR values in 9/11,82\%, and all had CRP values within the normal limits. Patients with endogenous endophthalmitis exhibited $\mathrm{CRP} \geq 10 \mathrm{mg} / \mathrm{L}$ in $5 / 7,71 \%$. 
Table 1 Erythrocyte sedimentation rate and C-reactive protein of patients with a first episode of active uveitis of unknown cause

\begin{tabular}{|c|c|c|c|c|c|c|c|}
\hline & \multirow[t]{2}{*}{ Total $N=174$} & \multicolumn{3}{|l|}{$\mathrm{ESR}^{\mathrm{a}}$} & \multicolumn{3}{|l|}{$\mathrm{CRP}^{\mathrm{a}}$} \\
\hline & & $<20(N=101)$ & $20-59(N=57)$ & $\geq 60(N=16)$ & $<10(N=131)$ & $10-59(N=33)$ & $\geq 60(N=10)$ \\
\hline Total & 174 & $101 / 174(58 \%)$ & $57 / 174(33 \%)$ & $16 / 174(9 \%)$ & $131 / 174(75 \%)$ & $33 / 174(19 \%)$ & $10 / 174(6 \%)$ \\
\hline Diabetes mellitus & $17 / 174(10 \%)$ & $5 / 17(29 \%)$ & $9 / 17(53 \%)$ & $3 / 17(18 \%)$ & $12 / 17(71 \%)$ & $5 / 17(29 \%)$ & 0 \\
\hline Immune suppressive medication $^{\mathrm{b}}$ & $17 / 174(10 \%)$ & $5 / 17(29 \%)$ & $7 / 17(41 \%)$ & $5 / 17(29 \%)$ & $12 / 17(71 \%)$ & $2 / 17(12 \%)$ & $3 / 17(18 \%)$ \\
\hline $\begin{array}{l}\text { Human immunodeficiency virus } \\
\text { positivity }^{c}\end{array}$ & $11 / 174(6 \%)$ & $2 / 11(18 \%)$ & $5 / 11(45 \%)$ & $4 / 11(36 \%)$ & 10/11 (91\%) & 0 & $1 / 11(9 \%)$ \\
\hline \multicolumn{8}{|l|}{ Anatomical localization } \\
\hline Anterior & $33 / 174(19 \%)$ & $18 / 33(55 \%)$ & $10 / 33(30 \%)$ & $5 / 33(15 \%)$ & $23 / 33(70 \%)$ & $8 / 33(24 \%)$ & $2 / 33(6 \%)$ \\
\hline Intermediate & $2 / 174(1 \%)$ & $1 / 2(50 \%)$ & $1(50 \%)$ & 0 & $2 / 2(100 \%)$ & 0 & 0 \\
\hline Posterior & $45 / 174(26 \%)$ & $32 / 45(71 \%)$ & $12 / 45(27 \%)$ & $1 / 45(2 \%)$ & $37 / 45(82 \%)$ & $8 / 45(18 \%)$ & 0 \\
\hline Panuveitis & $86 / 174(49 \%)$ & $48 / 86(56 \%)$ & $30 / 86(35 \%)$ & $8 / 86(9 \%)$ & $65 / 86(76 \%)$ & $15 / 86(17 \%)$ & $6 / 86(7 \%)$ \\
\hline Scleritis & $8 / 174(5 \%)$ & $2 / 8(25 \%)$ & $4 / 8(50 \%)$ & $2 / 8(25 \%)$ & $4 / 8(50 \%)$ & $2 / 8(25 \%)$ & $2 / 8(25 \%)$ \\
\hline Non-infectious systemic disease & $59 / 174(34 \%)$ & $33 / 59(56 \%)$ & $20 / 59(34 \%)$ & $6 / 59(10 \%)$ & $39 / 59(66 \%)$ & $14 / 59(24 \%)$ & $6 / 59(10 \%)$ \\
\hline Infectious uveitis & $38 / 174(22 \%)$ & $20 / 38(53 \%)$ & $11 / 38(29 \%)$ & $7 / 38(18 \%)$ & $31 / 38(82 \%)$ & $4 / 38(11 \%)$ & $3 / 38(8 \%)$ \\
\hline Established clinical entity & $24 / 174(14 \%)$ & $14 / 24(58 \%)$ & $8 / 24(33 \%)$ & $2 / 24(8 \%)$ & $17 / 24(71 \%)$ & $7 / 24(29 \%)$ & 0 \\
\hline Unknown & $53 / 174(30 \%)$ & $34 / 53(64 \%)$ & $18 / 53(34 \%)$ & $1 / 53(2 \%)$ & $44 / 53(83 \%)$ & $8 / 53(15 \%)$ & $1 / 53(2 \%)$ \\
\hline
\end{tabular}

ESR erythrocyte sedimentation rate, $C R P$ C-reactive protein

${ }^{\text {a }}$ ESR had to be determined $<2$ weeks of onset, CRP within $<1$ week of onset

${ }^{\mathrm{b}}$ Indicated for other causes than uveitis

${ }^{\mathrm{c}} \mathrm{HIV}$ was tested in 62 patients, out of which 11/62 (18\%) were found positive

Patients with non-infectious uveitis commonly had ESR and CRP values that were both within the normal limits $(28 / 59,47 \%$; Table 2). If elevated $(N=31)$, the parameters were most often elevated simultaneously $(15 / 31,48 \%)$. Of the patients with HLA B27-associated uveitis without systemic involvement, 2/8, 25\% exhibited CRP $\geq 10 \mathrm{mg} / \mathrm{L}$ and $1 / 8,13 \%$ had both $\mathrm{ESR} \geq 20 \mathrm{~mm} /$ $\mathrm{h}$ and CRP $\geq 10 \mathrm{mg} / \mathrm{L}$. Two patients had HLA B27-associated uveitis with systemic involvement of which one had high elevation of CRP $\geq 60 \mathrm{mg} / \mathrm{L}$ and ESR $\geq 20 \mathrm{~mm} / \mathrm{h}$ and the other exhibited normal values. Sarcoidosis-associated uveitis showed a predominantly elevated ESR $(13 / 24,54 \%$; in the range between

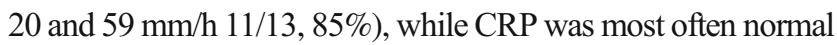
$(17 / 24,71 \%)$. Systemic involvement in sarcoidosis patients (hilar lymphadenopathy as seen on chest imaging) was present in 21/ 24 (88\%); however, in only 4/21 (19\%) of these, treatment was required.

The HIV was positive in $11 / 62,18 \%$ tested patients. HIVpositive patients had more often elevated ESR values when compared to the remainder of patients $(9 / 11,82 \%$ vs. $64 / 163$, $39 \%, P=0.009$, chi-square test). An infectious cause for uveitis was found in 9/11, 82\% HIV-positive patients, out of which $4 / 9,44 \%$ had CMV retinitis and $4 / 9,44 \%$ had syphilitic uveitis. ESR $\geq 60 \mathrm{~mm} / \mathrm{h}$ together with HIV positivity was observed in 4/11, 36\% (2 with CMV retinitis, one with syphilitic uveitis, and one with sarcoidosis-associated uveitis). Only one of the HIV-positive patients exhibited normal values of both ESR and CRP; this patient was diagnosed with CMV retinitis.

\section{Discussion}

In this retrospective, cross-sectional study, the majority of patients with a first active episode of uveitis of unknown origin presented with normal ESR and CRP values. Moreover, no significant relationship between the levels of these biomarkers and specific causes of uveitis was found.

Earlier investigations of ESR and CRP in uveitis patients also demonstrate normal values in a majority of patients with anterior uveitis, but none of these previous studies defined the time window in which ESR and CRP were determined in relation to the onset of uveitis, while both biomarkers are susceptible for changes within short periods [5, 8, 17]. Biomarkers like ESR and CRP are commonly assessed during the diagnostic workup of new uveitis patients for potential detection of infections or systemic immune-mediated disease-causing uveitis [1-4, 18-21]. Elevated levels of ESR are due to a higher plasma protein levels (e.g., fibrinogen, gamma globulins) and CRP is an acute phase protein released after tissue injury caused by infections or other sources of inflammation $[8,22]$.

Though a majority of patients with infectious uveitis in the present study exhibited normal ESR and CRP values, increased values were predominantly encountered in systemic infections. The high levels of ESR and CRP were found in patients with endogenous endophthalmitis, an ocular inflammation that occurs concurrently with bacteremia. In contrast, these inflammatory parameters were nearly always normal in 


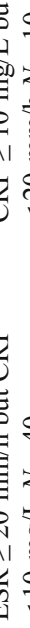

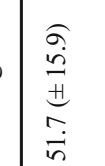

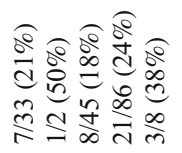

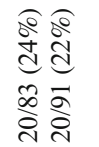

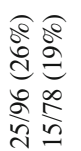

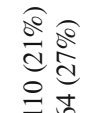

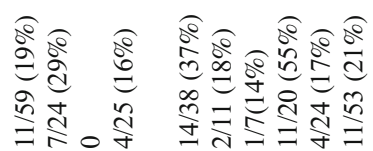

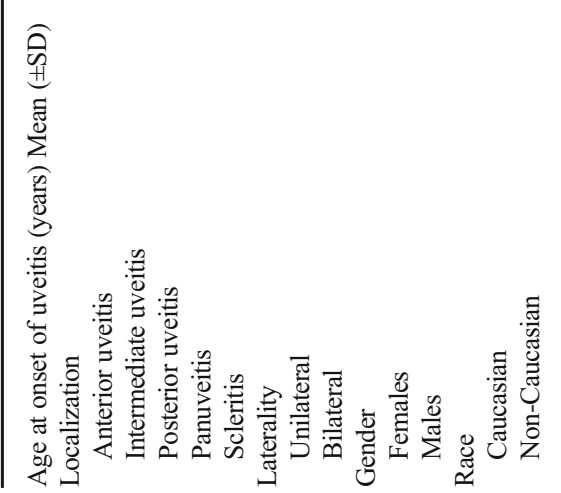

तิ

III

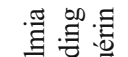

营

름.

독.

记

ㄴ.

青完

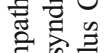

章 11

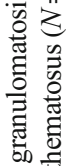

厐

II

总.

它

हैं

농

害:

๙ิ

当

तิ

¿.

零

兽焉

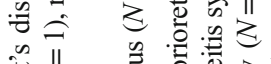

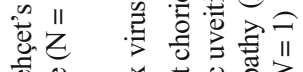

एँ

$\dot{f}: \stackrel{\mathscr{D}}{\Rightarrow}$

¿

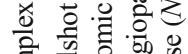

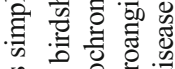

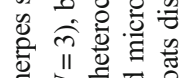

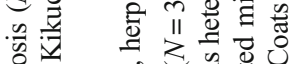

兽完

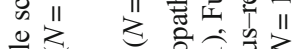
닐

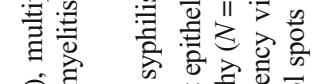
के 를 $\frac{0}{0}$ क

के

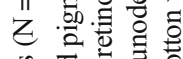

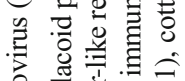

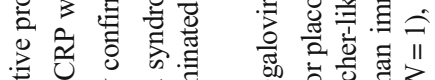

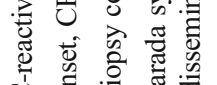

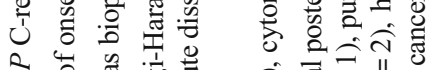

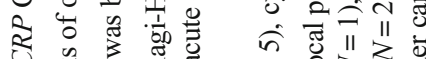

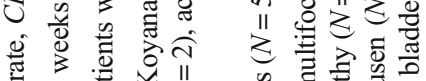

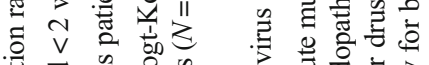

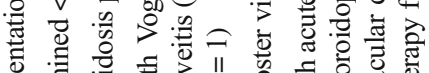

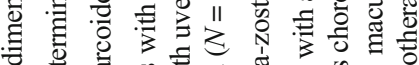

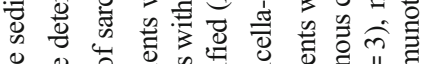

\% 0 पे

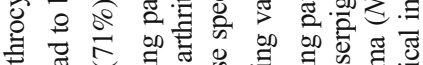

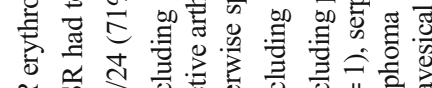

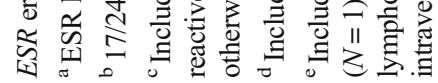


Table 3 Patients exhibiting highly elevated erythrocyte sedimentation rate and/or C-reactive protein

Erythrocyte sedimentation rate $\geq 60 \mathrm{~mm} / \mathrm{h}$ and/or C-reactive protein $\geq 60 \mathrm{mg} / \mathrm{L}(N=20)^{\mathrm{a}}$

$\begin{array}{cl}\text { Associated systemic } & \text { 2 Sarcoidosis } \\ \text { disease } & \text { 3 Reactive arthritis } \\ & \text { 1 Granulomatosis with polyangiitis, } \\ & \text { 1 Relapsing polychondritis } \\ & 1 \text { Behçet's disease } \\ \text { Infectious disease } & 3 \text { Endogenous endophthalmitis } \\ & \text { 2 Cytomegalovirus retinitis } \\ & 1 \text { Herpes simplex retinitis } \\ & 1 \text { Varicella-zoster anterior uveitis } \\ & 1 \text { Syphilis } \\ & 1 \text { Tuberculosis } \\ \text { Clinical entity } & 1 \text { Masquerade syndrome }{ }^{\mathrm{b}} \\ & 1 \text { Post-traumatic uveitis } \\ \text { Unknown } & 1^{\mathrm{b}}\end{array}$

a 10 patients had ESR $\geq 60 \mathrm{~mm} / \mathrm{h}, 4$ patients had CRP $\geq 60 \mathrm{mg} / \mathrm{L}$, and 6 patients both had ESR $\geq 60 \mathrm{~mm} / \mathrm{h}$ and CRP $\geq 60 \mathrm{mg} / \mathrm{L}$

${ }^{\mathrm{b}} 5$ patients with disease limited to the eye had some other explanation for high elevation of ESR and/or CRP, namely multiple myeloma complicated with varicella-zoster uveitis $(N=1)$, sepsis in herpes simplex-associated uveitis $(N=1)$, inflammatory bowel disease with masquerade syndrome of drusen $(N=1)$, post-operative infection after orthopedic surgery in a patient with post-traumatic uveitis $(N=1)$, and a patient with fever, cachexia, and uveitis of unknown origin $(N=1)$

patients with ocular toxoplasmosis, an intracellular parasite. Reactivation of these dormant parasites within the eye is not being accompanied by any systemic activity.

In HIV patients, the common hyperimmunoglobulinemia causes elevation of ESR (rather than a direct infectious trigger causing release of fibrinogen). In our series, highly elevated ESR was often seen in HIV-positive patients, and therefore, it might be therefore worthwhile to determine HIV status in patients with uveitis of unknown origin and unexplained high ESR [23]. Patients using immunosuppressive medications and those suffering from DM had more often elevated ESR values and the yield of these tests is therefore lower in these patients.

Elevated levels of ESR and CRP are common in systemic sarcoidosis patients, specifically in sarcoidosis-associated arthritis and erythema nodosum compared to other clinical presentations [24, 25]. Half of the patients with ocular sarcoidosis, however, had both normal ESR and CRP, which might reflect mild (or lack of) systemic involvement at the moment of onset of first uveitis attack [26]. This is also illustrated in the current series, where the majority of patients had very mild extraocular involvement which might also explain their predominantly normal ESR and CRP values.

In this study, follow-up measurements of ESR and/or CRP were not available, and consequently, we do not have information about the highest levels of ESR and CRP reached in individual patients. Including the highest levels of ESR and CRP could possibly expose some associations, which were not found in the present study. In addition, the changes of ESR and/or CRP might change in individual patients and might be associated with impeding uveitis activity. However, our main goal was to determine the diagnostic value of these parameters during the first stage of uveitis after presentation. Data on body mass index, which can influence the ESR and CRP values, were not available in our patients [27].

In conclusion, our study reflects that presence of uveitis alone is not sufficient to cause elevation of ESR and/or CRP as a majority of patients with a first uveitis episode had ESR and CRP values within the normal limits. In patients with highly elevated ESR and/or CRP, the presence of a systemic disease is very likely, and in consequence, the cause of uveitis is being established in a vast majority of cases.

Funding This research was supported by Abbvie Netherlands.

\section{Compliance with ethical standards}

Conflict of interest The authors declare that they have no conflict of interest.

Ethical approval All procedures performed in studies involving human participants were in accordance with the ethical standards of the institutional and/or national research committee and with the 1964 Helsinki declaration and its later amendments or comparable ethical standards. For this type of study (retrospective study), formal consent is not required.

Disclaimer The sponsor had no role in the design or conduct of this research.

Open Access This article is distributed under the terms of the Creative Commons Attribution 4.0 International License (http:// creativecommons.org/licenses/by/4.0/), which permits unrestricted use, distribution, and reproduction in any medium, provided you give appropriate credit to the original author(s) and the source, provide a link to the Creative Commons license, and indicate if changes were made.

\section{References}

1. Groen F, Ramdas W, de Hoog J et al (2016) Visual outcomes and ocular morbidity of patients with uveitis referred to a tertiary center during first year of follow-up. Eye (Lond) 30(3):473-480

2. Tsirouki T, Dastiridou A, Symeonidis C et al (2018) A focus on the epidemiology of uveitis. Ocul Immunol Inflamm 26(1):2-16

3. Miserocchi E, Fogliato G, Modorati G et al (2013) Review on the worldwide epidemiology of uveitis. Eur J Ophthalmol 23(5):705717

4. Acharya NR, Tham VM, Esterberg E et al (2013) Incidence and prevalence of uveitis results from the Pacific ocular inflammation study. JAMA Ophthalmol 131(11):1405-1412

5. Marsh JD, Markowitz BB (2012) Erythrocyte sedimentation rate and C-reactive protein in anterior uveitis Available from:http://iovs. arvojournals.org/article. aspx? articleid=2359946. [accessed 2017 24-08-2017]

6. Haasnoot AJW, van Tent-Hoeve M, Wulffraat NM et al (2015) Erythrocyte sedimentation rate as baseline predictor for the development of uveitis in children with juvenile idiopathic arthritis. Am J Ophthalmol 159(2):372-377 
7. von Elm E, Altman DG, Egger M et al (2008) The Strengthening the Reporting of Observational Studies in Epidemiology (STROBE) statement: guidelines for reporting observational studies. J Clin Epidemiol 61(4):344-349

8. Jain S, Gautam V, Naseem S (2011) Acute-phase proteins: as diagnostic tool. J Pharm Bioallied Sci 3(1):118-127

9. Jabs DA, Nussenblatt RB, Rosenbaum JT et al (2005) Standardization of uveitis nomenclature for reporting clinical data. Results of the First International Workshop. Am J Ophthalmol 140(3):509-516

10. Herbort CP, Rao NA, Mochizuki M et al (2009) International criteria for the diagnosis of ocular sarcoidosis: results of the first International Workshop on Ocular Sarcoidosis (IWOS). Ocul Immunol Inflamm 17(3):160-169

11. Rosenbaum JT (2017) New developments in uveitis associated with HLA B27. Curr Opin Rheumatol 29(4):298-303

12. Okada AA, Stanford M, Tabbara K (2012) Ancillary testing, diagnostic/classification criteria and severity grading in Behcet disease. Ocul Immunol Inflamm 20(6):387-393

13. Levinson RD, Brezin A, Rothova A et al (2006) Research criteria for the diagnosis of birdshot chorioretinopathy: results of an international consensus conference. Am J Ophthalmol 141(1):185-187

14. DeBoer JH, Verhagen C, Bruinenberg M et al (1996) Serologic and polymerase chain reaction analysis of intraocular fluids in the diagnosis of infectious uveitis. Am J Ophthalmol 121(6):650-658

15. Snyder DA, Tessler HH (1980) Vogt-Koyanagi-Harada syndrome. Am J Ophthalmol 90(1):69-75

16. Crawford CM, Igboeli O (2013) A review of the inflammatory chorioretinopathies: the white dot syndromes. ISRN Inflamm 2013:783190
17. Paul S (1963) Erythrocyte sedimentation rate in uveitis - a critical analysis. Indian J Ophthalmol 11(1):9-12

18. Hooper C, McCluskey P (2008) Intraocular inflammation: its causes and investigations. Curr Allergy Asthma Rep 8(4):331-338

19. Rosenbaum JT, Wernick R (1990) Selection and interpretation of laboratory tests for patients with uveitis. Int Ophthalmol Clin 30(4): 238-243

20. Rosenbaum JT (1990) An algorithm for the systemic evaluation of patients with uveitis: guidelines for the consultant. Semin Arthritis Rheum 19(4):248-257

21. Wade NK, Kevin MD, F.R.C.P.C. et al (2000) Diagnostic testing in patients with ocular inflammation. Int Ophthalmol Clin 40(2):3754

22. Pepys MB, Hirschfield GM (2003) C-reactive protein: a critical update (vol 111, pg 1805, 2003). J Clin Investig 112(2):299-299

23. Lowe DM (2010) The erythrocyte sedimentation rate in HIV: a neglected parameter? AIDS 24(18):2773-2775

24. Shorr AF et al (2000) Osseous disease in patients with pulmonary sarcoidosis and musculoskeletal symptoms. Respir Med 94(3):228232

25. Greenberg G, James DG, Feizi T et al (1964) Serum-proteins in sarcoidosis. Lancet 2(7373):1313-1315

26. Edelsten C, Pearson A, Joynes E et al (1999) The ocular and systemic prognosis of patients presenting with sarcoid uveitis. Eye 13: 748-753

27. Samocha-Bonet D, Lichtenberg D, Tomer A et al (2003) Enhanced erythrocyte adhesiveness/aggregation in obesity corresponds to low-grade inflammation. Obes Res 11(3):403-407 